Cahiers d'études africaines

169-170|2003

Enseignements

\title{
Les travers du savoir
}

Représentations du diplôme et du travail au Maroc

\section{Étienne Gérard et Bernard Schlemmer}

\section{(2) OpenEdition}

Journals

Édition électronique

URL : http://journals.openedition.org/etudesafricaines/201

DOI : 10.4000/etudesafricaines.201

ISSN : 1777-5353

Éditeur

Éditions de l'EHESS

Édition imprimée

Date de publication : 1 janvier 2003

Pagination : 299-319

ISBN : 978-2-7132-1809-5

ISSN : 0008-0055

Référence électronique

Étienne Gérard et Bernard Schlemmer, «Les travers du savoir », Cahiers d'études africaines [En ligne], 169-170 | 2003, mis en ligne le 21 décembre 2006, consulté le 03 mai 2019. URL : http:// journals.openedition.org/etudesafricaines/201 ; DOI : 10.4000/etudesafricaines.201

(c) Cahiers d'Études africaines 


\title{
Étienne Gérard et Bernard Schlemmer
}

\author{
Les travers du savoir \\ Représentations du diplôme \\ et du travail au Maroc
}

Le chômage intellectuel frappe de nombreux pays, et en particulier l'Afrique où «non seulement le diplôme n'est plus un viatique contre le chômage, mais il est même systématiquement devenu un facteur de risque pour trouver un emploi » (Antoine et al. 2001: 32). Au Maroc, en 1997, 30,5\% des diplômés de l'enseignement supérieur de milieu urbain étaient à la recherche d'un emploi, contre 9,6\% des non diplômés, et 69 \% des chômeurs avaient un diplôme moyen ou supérieur (Direction de la statistique 1999) ${ }^{1}$. Parallèlement, le taux de chômage des diplômés est passé de $26,5 \%$ à $27,5 \%$ entre le $1^{\text {er }}$ et le $2^{\mathrm{e}}$ trimestre de l'année $2000^{2}$.

«Au Maroc, on ne parle de chômage que pour les étudiants », observe avec justesse un jeune diplômé interviewé. Combinée avec les réflexions et les politiques menées autour de «l'adéquation formation-emploi », une telle assertion met en exergue le rapport communément établi entre université et monde du travail et invite à s'interroger sur le «statut» du savoir (en l'occurrence universitaire) dans la société, en particulier aux yeux des premiers concernés, les diplômés ${ }^{3}$. Le discours libéral dominant, en effet, établit ou entérine une équation entre études et travail, évaluant les unes à l'aune de l'autre. Dans quelle mesure une telle perspective façonne-t-elle les représentations du savoir universitaire ? Parallèlement, quels peuvent

1. Al Bayane, 24/10/2000.

2. La durée moyenne de chômage est de 39,5 mois pour la population sans diplôme, 43,1 mois pour les titulaires d'un diplôme de niveau moyen, et 41,6 mois pour ceux qui ont un niveau supérieur au baccalauréat (Direction de la statistique 1999, ministère du Plan, Rabat).

3. La catégorie de "diplômés-chômeurs », politiquement et médiatiquement construite, représente, selon les estimations, plus de 200000 individus (à titre indicatif, 192056 étudiants sont sortis de l'enseignement supérieur en 1990, 266507 en 1998). Le terme de "diplômés-chômeurs » est bien sûr paradoxal : il réfère en effet à la banalisation d'une situation (le chômage des diplômés) et à l'originalité du substrat de cette situation (le diplôme) (GEISSER 2000 : 19-30). 
être les effets de l'évolution de l'université - au-delà d'une simple inadéquation avec le marché de l'emploi - sur les conceptions du savoir et sur les modes actuels de son «utilisation » par ses détenteurs ? Pour aborder ces questions, nous nous intéresserons en premier lieu à l'histoire de l'enseignement et de l'université, et au statut du diplôme dans cette phase où il donnait systématiquement accès à l'emploi. Nous nous arrêterons ensuite sur les discours de jeunes diplômés de milieu populaire, aujourd'hui pris dans une situation inverse, avant d'examiner quelques rapports entre les représentations du diplôme, du savoir et du travail que révèle l'évolution du Maroc.

\section{La construction historique d'un système d'enseignement}

Quand le Maroc accède à l'indépendance, en 1956, le dessein historique assigné au nouveau gouvernement se résout en quatre principes de base : arabisation (progressivement, l'arabe doit devenir la seule langue d'enseignement), marocanisation (les Marocains doivent rapidement remplacer les coopérants), unification (le système doit devenir homogène, sous le contrôle de l'État $^{4}$ ) et généralisation (dans les meilleurs délais, un enseignement de base doit être reçu par tous les Marocains, et l'accès à l'éducation doit être ouvert à tous). Toute la question était alors : entre pragmatisme et volontarisme, quel équilibre fallait-il tenir, quel rythme fallait-il adopter ?

Paradoxalement, le problème se posait avec d'autant plus d'acuité que le pays ne partait pas d'une tabula rasa, même en ce qui concernait l'enseignement supérieur. Avant le Protectorat, de nombreuses médersas formaient les élites du pays, dispensant un enseignement certes essentiellement théologique et juridique, mais également parfois médical et scientifique ; surtout, le Maroc pouvait se glorifier d'abriter, avec la Qaraouiyyîn, une université qui, du XIII ${ }^{\mathrm{e}}$ au XV $\mathrm{XV}^{\mathrm{e}}$ siècle, compta parmi les plus prestigieuses du monde, et pas seulement du monde musulman. Avec le repli sur son identité religieuse et sa fermeture à tout ce qui était perçu comme hétérogène à la tradition théologique (ce qui lui interdisait l'étude de toutes les disciplines scientifiques), elle perdit de son aura. «C'est pourquoi elle n'eut pas la même "renaissance" que certaines universités de l'Orient arabe à la fin du $\mathrm{XIX}^{\mathrm{e}}$ et au début du XX $\mathrm{XX}^{\mathrm{e}}$ siècle » (Baina 1981: 95). Elle ne constituait pas moins un pôle de référence de l'identité marocaine et un lieu de haut savoir

4. Sous le Protectorat français, on comptait trois, voire quatre systèmes conçus, au départ, comme étanches : l'enseignement musulman traditionnel, l'enseignement public destiné aux musulmans, l'enseignement public destiné aux Européens, auquel il convient d'ajouter l'enseignement musulman « rénové », écoles privées mises en place par des nationalistes. Cela aurait pu constituer un handicap majeur, mais « les quatre principes d'unification, de marocanisation, d'arabisation et de généralisation avaient tellement imprégné l'enseignement marocain que les écoles privées impulsées par les partis nationalistes, qui étaient légion sous le Protectorat [...], se sont fondus dans l'ensemble qui était espéré » (ANONYME $1985: 25$ ). 
qui allait fournir au pays une élite intellectuelle reconnue et rester jusqu'à aujourd'hui la figure centrale de l'enseignement originel.

À l'indépendance, les attentes étaient particulièrement fortes : avec les nationalistes qui avaient ouvert le chemin d'un enseignement musulman rénové, une part importante de la population était déjà acquise à un enseignement moderne. Plus encore, entre l'élite traditionnelle, qui sortait de la Qaraouiyyîn, et le plus pauvre paysan, qui connaissait le chemin du $m s \hat{\imath} d^{5}$, c'est l'ensemble de la population qui, de longue date déjà, était familiarisée, sinon avec l'éducation de type occidental, du moins avec l'idée que l'éducation de l'enfant pourrait se voir confiée à une autre autorité que la famille — ce qui est la base même de l'institution scolaire ${ }^{6}$. Une infime partie ${ }^{7}$ accédait même à l'enseignement supérieur, en lettres (à l'Institut des hautes études marocaines, au Centre d'études juridiques, ou à l'École marocaine d'administration) ou en sciences (à l'Institut chérifien ou à l'École d'agriculture).

\section{Du côté de l'offre...}

On comprend que la priorité ait été donnée à la mise en place des infrastructures, dramatiquement insuffisantes. "La question de la création d'une Université nationale se posait, les uns estimant que l'enseignement supérieur, étant trop coûteux, n'avait pas à être fait au Maroc, les autres, qu'il fallait développer le cadre existant, en le nationalisant, afin de répondre aux besoins croissants du pays » (Ben Messaoud 1970). C'est cette thèse qui prévalut et, progressivement, furent mis en place les établissements nécessaires pour former, dans le pays, des cadres maîtrisant les principales technologies indispensables au Maroc.

Mais le ministère de l'Éducation nationale, surtout soucieux de développer l'enseignement primaire, ne se consacre alors guère à l'université, pour laquelle il ne semble pas suivre de plan précis. L'urgent n'est pas la qualité, mais la quantité, et ce n'est pas dans les établissements supérieurs que les difficultés se présentent : là du moins, on trouve, par le biais de la coopération, des enseignants qualifiés et des élèves qui, déjà formés en français par les écoles du Protectorat, n'ont pas de difficultés particulières à suivre un enseignement qui prolonge, sous le même mode, celui qu'ils ont connu dans le secondaire. Certes, l'État ne se désintéresse pas totalement de la

5. École coranique de base. À la veille du Protectorat, on estime qu'un enfant sur six l'avait fréquentée (JENAISTAR 1980 : 55).

6. En outre, «beaucoup de parents ont une stratégie scolaire bivalente : un peu de $m s \hat{\imath} d$, puis un peu d'école du rûmî [l'Européen]. Comme s'ils voulaient préserver les chances de réussite sociale de leurs enfants dans ce bas monde et assurer le salut de ceux-ci dans le monde de l'au-delà » (RIVET 1999 : 278).

7. En 1955, on compte, en fait de «cadres musulmans formés pendant la période du protectorat : 30 ingénieurs, 19 médecins, 2 dentistes, 27 avocats et 6 pharmaciens. Sur 5500 hauts et moyens fonctionnaires dans l'administration du Protectorat, on dénombre 165 Marocains » (Souali \& Merrouni 1981: 20). 
formation d'une élite compétente dans les divers secteurs dont il aura besoin, et met peu à peu en place des établissements d'enseignement. Mais — au moins jusqu'en 1973 — on ne cherchera pas véritablement à se donner les moyens de pratiquer une politique universitaire. De même, on ne se souciera guère, concrètement, d'améliorer la pédagogie des enseignements ${ }^{8}$ ou d'orienter les élèves ; on ne cherchera pas non plus à déterminer le nombre d'étudiants souhaitable (que ce soit en chiffres absolus ou selon les branches), ni à se donner les moyens d'orienter les choix en fonction du marché du travail et des besoins du pays. C'est au primaire, d'abord, que le mot d'ordre de généralisation doit s'appliquer, et dans le primaire et le secondaire que marocanisation et arabisation semblent plus ou moins rapidement réalisables. Quant au mot d'ordre d'unification, si l'on peut dire qu'il s'est réalisé, il faudrait préciser que l'État marocain a mis en place, pour l'enseignement supérieur, un très étrange système ${ }^{9}$, dans lequel facultés ou instituts rattachés à des universités dépendent du ministère de l'Éducation nationale, mais où toutes les autres grandes écoles et instituts, qui bénéficient d'un recrutement sur concours et d'un taux d'encadrement bien supérieur, sont sous tutelle des différents ministères auxquels ils correspondent. Le ministère de l'Éducation nationale perd ainsi tout contrôle efficace sur des établissements scientifiques les plus prestigieux.

Il faudra attendre une décennie pour qu' on réalise le danger, et encore : «En 1966, les tentatives de la "Nouvelle Doctrine" 10 pour réformer l'enseignement concernaient le premier et le second degré, se contentant de prévoir la gravité des problèmes qu'allait connaître l'enseignement supérieur si la ligne politique n'était pas révisée » (Souali 1983b: 29). Mais le pouvoir n'a pas d'autre politique que de tenter d'obtenir une « maîtrise des flux » ${ }^{11}$,

8. Les différents plans d'amélioration de l'enseignement insisteront tous sur la nécessaire amélioration de la qualification des maîtres, certes ; mais le diplôme est devenu un tel enjeu que l'apprentissage par cœur de cours mal maîtrisés est un leitmotiv constant dans le discours des enseignants eux-mêmes.

9. Inspiré du système français, où coexistent grandes écoles élitistes et universités ouvertes à tous.

10. La « Nouvelle doctrine » est le terme consacré pour désigner la politique proposée par le ministre de l'Éducation nationale d'alors, le Dr Benhima. Celui-ci, critiquant implicitement le volontarisme antérieur, préconisait la poursuite de l'effort de généralisation dans le primaire, mais la sélection à l'entrée du secondaire et une réglementation sévère des redoublements, le maintien et le retour $\mathrm{du}$ français comme langue d'enseignement dans les matières scientifiques arabisées, un relèvement du niveau des cadres enseignants (fût-ce aux dépens de la marocanisation), et un développement de l'enseignement scientifique et technique. Ce programme «ne fut plus endossé par les ministres suivants ni adopté explicitement par le gouvernement. Il est resté, néanmoins, le référant implicite de l'action gouvernementale» (SouAli 1983a).

11. «Ne pouvant aborder de front la maîtrise des flux à l'entrée de l'université, l'action du gouvernement [...] a pu se concentrer autour de trois lignes d'efforts principales : continuer d'étendre les capacités d'accueil, accélérer les écoulements et la fluidité à l'intérieur de l'Université, agir sur l'amont pour diminuer la pression à l'entrée du supérieur » (EL MassLOUT 1999: 42-43). 
avec « la tentation, en période normale, de geler les problèmes et les situations et d'adopter des solutions de facilité et, en période de crise, le refus de répondre à des revendications qu'on sait ne pouvoir satisfaire, donc la recherche de boucs émissaires et, en fin de compte, l'appel lancé au ministère de l'Intérieur pour qu'il résolve les problèmes du ministère de l'Éducation nationale » (Ben Messaoud 1970 : 53). Le pays connaîtra, tout au long des années 1968-1973, puis de 1978 à 1981, une période extrêmement dure en termes de luttes étudiantes (lato sensu) et de répression policière. Ainsi, pendant plus de trente ans, «les mesures prises pour modifier certains aspects du système éducatif le seront sous la pression des événements, sanctionnant une réalité de fait et non l'application d'une réflexion prospective » (Souali 1983a : 32-33).

Ne parlons même pas, dans ces conditions, de politique de science : l'effort financier consenti à la recherche n'a jamais été à la hauteur des ambitions parfois proclamées, et, bien au contraire, les enseignants-chercheurs ont été contraints de faire face à des conditions de travail telles que toute recherche personnelle, voire tout encadrement de recherche, relevait du sacerdoce (Ben Messaoud 1970). Il faudra attendre la réforme de 1975 pour voir mis en place un statut de l'enseignant-chercheur, et celle de 1997 pour que les avancements de carrière tiennent compte des publications.

\section{... Et du côté de la demande}

Durant les premières années de l'indépendance, il existe une concordance entre la demande et l'offre de formation, en lettres comme en sciences. Au départ, l'État propose un emploi à tous les diplômés ${ }^{12}$. L'emploi étant dans la fonction publique, on comprend qu'une large majorité des étudiants choisisse de préférence les études juridiques ${ }^{13}$, qui leur offrent de meilleures perspectives d'avancement de carrière dans des postes à responsabilité ${ }^{14}$. Mais le goût pour les études scientifiques n'interdit pas de rêver à ces emplois, auxquels le diplôme, de par le seul fait qu'il soit issu d'études universitaires, semble ouvrir un droit. «Les ingénieurs au Maroc sont des salariés de l'État», nous rappelle P. Pascon (1977: 22), qui poursuit: «Généralement, l'ingénieur dans un pays dominant trouve place dans une spécialité plus étroite que celle pour laquelle il a été formé, il approfondit

12. En 1983, près de $78 \%$ des ingénieurs sont employés par l'État, et 78,1\% d'entre eux sont des ingénieurs d'études et non des ingénieurs d'État (c'est-à-dire destinés à l'application plus qu'à la recherche). Précisons encore que $35 \%$ sont des ingénieurs agricoles (SALMI 1984).

13. «En 1976-1977, sur 46858 étudiants inscrits dans l'enseignement supérieur, 23731 [soit plus de la moitié] étaient inscrits dans les facultés de Droit » (OuAhab \& NoRedDine 1980: 47).

14. «Dans le monde arabe, le haut cadre est structurellement du pouvoir politique » (Khatibi $1980: 35$ ). 
sa technique, il resserre le champ de ses intérêts avant de voir les choses plus largement. Sauf exception, dans nos pays, l'ingénieur fonctionnaire d'État est placé à un poste peu en rapport avec la spécialité qu'il a choisie et d'emblée avec des responsabilités qui dépassent très largement l'expérience qu'il a pu en avoir » (ibid. : 33).

Dès les années 1970 cependant, « la société marocaine est redevenue une société bloquée [...]. Les anciens intermédiaires du protectorat sont devenus dirigeants et une nouvelle classe bureaucratique s'est formée pour prendre les places d'intermédiaires devenues vacantes. Nous sommes revenus à une situation de quasi-rigidité sociale » (Mounir 1972: 18). La population étudiante, représentée par l'Union nationale des étudiants marocains (UNEM), mettra elle aussi un certain temps avant d'en prendre conscience. Dans les tout premiers temps de l'indépendance, elle aura apporté un soutien critique au nouveau régime - encore perçu comme l'expression du mouvement national. Dès les années 1960, cependant, avec les nouveaux clivages politiques à la tête de l'État et l'éviction des partis de gauche, elle entrera dans une opposition radicale. Son exigence, si elle porte d'abord sur la liberté politique et la démocratisation (de l'enseignement, mais elle passe, à ses yeux, par celle du régime), porte aussi, tout comme celle des organisations politiques et syndicales (Istiqlal, UNFP, UMT, SNES...), sur un volontarisme accru dans la politique d'arabisation, dont cette génération éduquée en français n'a objectivement pas besoin pour acquérir un savoir qualifié, et dont elle ne tire aucun avantage en termes de valorisation de son diplôme.

Il faudra attendre "l'émergence d'une population étudiante provenant davantage des couches déshéritées du pays, ayant mis tous ses espoirs dans l'éducation comme instrument de promotion et de mobilité et voyant ces espoirs menacés, sinon anéantis » (Ben Messaoud 1970: 53) pour que la situation se modifie. Cela ne se traduit pas tant par un changement du discours des organisations étudiantes que par une désaffection vis-à-vis de ces syndicats. Après la levée de son interdiction ${ }^{15}$, l'UNEM, en particulier, n'a pas évolué comme la population étudiante, n'a pas su prendre à bras le corps la gestion quotidienne, concrète, de ses problèmes. Elle y perdra d'abord sa capacité à intéresser une majorité d'étudiants, puis le leadership dans les universités, jusqu'à laisser la place, ces dernières années, aux courants religieux. Il est possible que ceux-ci ne fassent partager leurs convictions révolutionnaires ${ }^{16}$ qu'à une minorité de la population estudiantine, mais ils occupent désormais pratiquement seuls le terrain ${ }^{17} \ldots$ « L'Université marocaine est passée par trois grandes étapes. D’abord les années 1960/1970

15. Prononcée en 1973, elle ne sera levée qu'en 1979.

16. Convictions religieuses, et non plus progressistes et laïques, mais néanmoins « révolutionnaires » puisque proposant un changement en profondeur du système établi, et donc capable d'une force de conviction réelle.

17. Il faudra attendre les années 2000 pour voir décliner quelque peu sinon l'aura — difficile à mesurer - du moins l'importance des mouvements islamiques dans les universités marocaines. 
où les étudiants représentaient une pépinière. L'engagement politique était d'abord l'expression d'une citoyenneté qui se faisait à travers l'adhésion à un parti de l'opposition. Il fallait se battre pour conquérir les libertés. Puis il y eut les années 1980, les années de la transition chaotique. Les années 1990 ont généré des étudiants qui n'avaient plus d'idéal sinon celui de rechercher un emploi », explique froidement Mustapha Zaoui (cité dans Rerhaye 2001 : 11).

\section{Sic itur ad astra}

Garantie d'accès à l'emploi, le diplôme universitaire - même scientifique - a servi au départ à mettre le pied à l'étrier dans une carrière qui ne serait pas forcément scientifique, mais qui serait au service de la nation. Quand ce modèle a commencé à ne plus fonctionner, une partie des étudiants a cherché à suivre des cursus qui offraient les meilleurs débouchés, et l'on a assisté à un certain rééquilibrage entre disciplines scientifiques et disciplines littéraires. Mais c'est surtout vers les grandes écoles que se dirigent maintenant les étudiants des disciplines scientifiques — du moins ceux à qui le capital scolaire, social et économique permet une telle orientation. Pour les autres, il est frappant de voir combien l'attente vis-à-vis de l'État, censé pourvoir aux besoins de ses enfants méritants, est restée forte même si, objectivement et manifestement, cette attente s'est avérée vaine.

Suffit-il, pour l'expliquer, de rappeler que « l'élite intellectuelle fait partie du système, même si elle n'est pas d'accord avec le pouvoir et la politique : d'une manière ou d'une autre, elle parle le même langage que lui, et raisonne à l'intérieur du même cadre de pensée, même et surtout quand elle manifeste à son égard une contre-dépendance profonde. En fait, elle partage les mêmes valeurs fondamentales, les mêmes références, et spécialement celle-ci : croyance en l'État et en sa toute puissance comme levier de développement et sujet de l'histoire » (Idrissi 1981: 4). Nous pensons plutôt qu'une véritable rupture qualitative est en cours dans la perception du diplôme, voire du savoir universitaire. Les premières générations qui ont suivi l'indépendance ont connu une phase historique exceptionnelle, où les théories de la reproduction des élites par l'université ne s'appliquaient que de manière très marginale et où, au contraire, l'enseignement représentait réellement une voie royale de promotion sociale. Certes, les grandes familles marocaines sont toujours en place, et les élites anciennes se retrouvent encore aux postes de commandes. Mais à côté, une part non négligeable de l'appareil d'État et des hauts responsables de l'économie marocaine est issue de familles rurales, populaires ${ }^{18}$.

18. En particulier, on notera que, malgré la répression à laquelle a dû faire face le syndicalisme étudiant, l'UNEM a toujours constitué une pépinière pour la formation des dirigeants. 
Ce n'est pas tant que «le diplôme » était, alors, hautement valorisé : la question ne se posait même pas, il ouvrait automatiquement à un poste à responsabilité, et le plus souvent dans la fonction publique. Ce n'est pas nécessairement le savoir, non plus, qui était recherché, puisque la carrière n'avait pas forcément de rapport avec la formation reçue. Mais la voie de l'enseignement supérieur, elle, s'imposait d'évidence comme la voie du progrès et du développement. Il ne restait qu'à la faire fonctionner au mieux... d'où les critiques croissantes adressées à un système universitaire qui peinait de plus en plus à remplir cette mission ${ }^{19}$.

Nous verrons qu'il en est tout autrement pour la génération actuelle y compris, sans doute, pour ceux qui trouvent rapidement leur place sur le marché du travail. Le rôle d'ascenseur social que jouait efficacement l'École commence à se gripper dans les années 1970, pour finir par se bloquer totalement dans les années 1990. Désormais, la voie de l'enseignement supérieur a révélé toutes ses faiblesses, en particulier son incapacité à assurer plus avant la mobilité sociale et l'accès des classes populaires aux postes de décision. Que vaut alors le savoir acquis, et que représente le diplôme universitaire, ailleurs que sur le marché de l'emploi où il se révèle si dévalué aux yeux des jeunes de milieu populaire qui l'ont acquis? Que faire d'un rêve brisé ?

\section{Les voies du cursus universitaire}

En raison de la rareté des emplois offerts par l'État (consécutive, pour partie, aux Plans d'ajustement structurel), la valeur des diplômes sur le marché du travail s'est dépréciée (Ibaaquil 1990) : pour les jeunes issus de la faculté, la licence ${ }^{20}$ constitue aujourd'hui l'indice minimal d'embauche dans le secteur privé, le niveau à atteindre pour espérer trouver un travail « décent », le seuil en deçà duquel l'emploi et sa rémunération répondent à l'arbitraire, dans l'ignorance du savoir acquis.

19. Parlant du Protectorat, D. Rivet (1999: 279) écrit : «Entre les techniciens de la machine scolaire du Protectorat et les parents, on nage dans le malentendu. Pour ceux-ci, l'école du rûmî a pour raison de former des fonctionnaires, de manière à pourvoir leur progéniture d'une place (blaça) au sein du Makhzen. Pour ceux-là, l'école moderne a pour finalité de fournir à la colonisation une élite de chefs de culture dans le bled, de contremaîtres sur les chantiers, d'ouvriers professionnels en usine, d'employés dans les établissements de commerce, et de secrétaires dans l'administration: bref, une cascade d'intermédiaires depuis le haut Makhzen jusqu'à la ferme du colon et au carreau de la mine. » Ce même malentendu se reproduit, à partir des années 1970, entre le peuple et l'État marocain et perdure encore, malgré l'argumentaire libéral que lui oppose la classe dirigeante.

20. Au Maroc, la licence est obtenue après quatre années de faculté. Elle correspond à la maîtrise française. 
Pour la plupart des licenciés interrogés ${ }^{21}$, ce n'est pas le savoir qu'ils recherchaient en priorité en entrant à l'université. "Tout le monde fait des études pour avoir un emploi. Personne ne fait des études pour les études. Ça, c'était avant, pas aujourd'hui », explique Noureddine, licencié en chimie en 1996. Faute de capital social ${ }^{22}$ et économique ou faute d'avoir obtenu des notes suffisantes, ils n'ont simplement pas pu prétendre entrer dans un autre cycle de formation supérieur, comme une école d'ingénieur. Sitôt l'examen obtenu, le bachelier passe donc des concours ${ }^{23}$ (de commissaires, instituteurs, gardiens de la paix), pour entrer dans la fonction publique... tout en s'inscrivant à l'université. "Parce que c'était la seule porte ouverte », explique par exemple Abdeltif (DEUG de biologie, 1994) «c'est tout ce qui me restait [...]. Car, si on n'entre pas à l'université, on perd tout. » Le milieu familial est là, aussi, qui pousse à la poursuite des études. Fils ou filles élus parmi leurs frères et sœurs ${ }^{24}$, «tes parents t'obligent à faire l'impossible, parce qu'ils ont fait beaucoup pour toi, ils ont fourni de l'argent, beaucoup de choses », relève par exemple Omar, licencié de géographie en 1996.

Alors que tout diplômé pouvait obtenir une intégration dans la fonction publique, l'État exige désormais de lui qu'il ait, au minimum, le baccalauréat, et le secteur privé ne recrute plus de cadre qu'au niveau de la licence.

$\mathrm{Au}$ désir de poursuivre des études poussées, dans l'espoir d'obtenir un travail qui soit à la hauteur de celles-ci, succède facilement la volonté première de ne pas être «sans rien » et de ne pas subir la loi arbitraire du marché du travail. L'entrée à l'université est ainsi le plus souvent le résultat d'un choix par défaut. Reculer le plus loin possible l'entrée dans l'inactivité s'offre alors comme la meilleure alternative ${ }^{25}$. Les aînés au chômage, occupés à travers des grèves de la faim à revendiquer une embauche par

21. Tous les éléments relatifs ici aux jeunes diplômés de l'université — à leurs situations, représentations et pratiques —, résultent d'une enquête réalisée dans la région de Fès, de février à mai 2001, auprès d'une trentaine d'entre eux, par entretiens semi-directifs. Tous sont de milieu populaire, parfois très pauvre (tel $\mathrm{N}$., dont la famille, trop nombreuse, se partage l'espace exigu de la seule pièce disponible : certains dorment la nuit, d'autres prennent leur place le jour).

22. Le capital social permet de contourner les barrières de la compétition universitaire. "Au Maroc», dit par exemple Idrissi, titulaire d'une licence de biologie depuis 1997, "il n'y a pas de concours, seulement des coups de piston. Les concours c'est une façade, comme un foulard pour calmer les étudiants, pour masquer les choses, empêcher de les voir. »

23. C'est le cas pour la très grande majorité des jeunes interviewés. En outre, une fois la licence obtenue, ils repassent des concours d'entrée dans la fonction publique et cherchent à compléter leurs études universitaires par une formation plus « qualifiante » (reconnue sur le marché de l'emploi), comme en informatique.

24. La plupart de ceux que nous avons rencontrés sont seuls, dans leur fratrie, à avoir fait des études supérieures.

25. Certains jeunes rencontrés ont ainsi passé sept ans à la faculté pour obtenir leur licence et n'ont interrompu leurs études qu'une fois ce titre obtenu. Cette possibilité est néanmoins aujourd'hui entravée par l'interdiction de redoubler plus d'une fois dans chaque cycle universitaire. 
l'État, montrent combien l'inactivité — « le chômage des diplômés » — est désormais le lieu commun des titulaires du baccalauréat et de la licence, et le stigmate auquel recourt ou se réfère tout un chacun pour parler d'eux. Face à ce stigmate, la valeur du diplôme est, aux yeux mêmes de ces lauréats, relative, et le sentiment d'exclusion ou de marginalité, consécutif au chômage, est, lui aussi, inégalement ressenti et interprété.

\section{Ceux qui maintiennent un projet scientifique}

Saïd, qui est diplômé en statistiques, ou Najib, qui a obtenu une licence d'électronique, considèrent que «le diplôme est un mérite » et qu'il leur faut mobiliser toutes les ressources possibles pour le valoriser. Saïd s'est vu privé de la possibilité de passer le concours de l'agrégation, supprimé au terme de son année de préparation. Des cours particuliers de mathématiques, de physique, ou encore de langue arabe dans une petite école privée tenue par un de ses voisins lui permettent tout juste de vivre. Après le baccalauréat, Najib a passé des concours pour entrer en classe préparatoire, à l'Institut agronomique et vétérinaire, et dans l'école de formation d'aviateurs. Après sa licence, il avait lui aussi tenté l'agrégation, supprimée depuis. La lecture d'ouvrages de physique, venus de l'étranger, entretient sa volonté de poursuivre en $3^{\mathrm{e}}$ cycle, au Canada, en Belgique ou en France, où il a déjà fait parvenir des dossiers d'inscription. Saïd était entré à l'université « pour devenir un grand chercheur » et a choisi l'étude des statistiques parce que c'était «un monde nouveau, le monde de la recherche». Najib avait pour objectif «d'être un savant... C'est cette ambition qui m'oriente toujours ». Malgré les handicaps propres au marché universitaire, l'un et l'autre n'ont pas renoncé à poursuivre jusqu' au doctorat. La vacance obligatoire jusqu'à obtention d'une inscription en $3^{\mathrm{e}}$ cycle n'en est pas vraiment une : tout le temps est consacré à la recherche des capitaux — argent, mais aussi livres et documents, relations à l'étranger - nécessaires à l'obtention du titre universitaire le plus élevé, indispensable à l'exercice d'un travail en lien direct avec les connaissances acquises.

Pour eux, en un sens, le savoir a des raisons que le travail ne connaît pas : au-delà, ou en deçà de la position qu'il occuperait dans la société, il possède une valeur intrinsèque, qui justifie la quête et l'exigence d'une reconnaissance, même symbolique. Toutes les voies d'enrichissement et de valorisation du savoir sont examinées, inventoriées, tous les capitaux sont mobilisés, qu'ils soient à disposition dans le cercle familial (argent, relations au Maroc ou à l'étranger) ou dans l'espace public (cybercafés où l'internet permet d'accéder aux facultés à l'étranger, Centres d'information et d'orientation pour l'emploi ${ }^{26}$, offres de stages et ouvertures de concours dans la

26. Les Centres d'information et d'orientation pour l'emploi (CIOPE), créés en 1993 par le ministère de l'Emploi (10 au départ, au nombre de 22 aujourd'hui), ont pour vocation de favoriser l'auto-emploi et l'insertion professionnelle des jeunes diplômés en leur offrant des stages en entreprise pour 18 mois (contrat formation insertion). 
fonction publique...). Et la conscience d'être « savant» — associée au sentiment d'une certaine supériorité — dans un milieu où le travail fait loi, pallie ce que d'autres ressentent comme une marginalisation : car le savoir définit une place nécessairement distincte et compose une identité marquée par la différence. La croyance dans la supériorité intrinsèque du savoir — c'est par lui, et non par le travail, que l'homme se réalise en premier, c'est lui qui confère son sens au travail - légitime la possibilité d'accéder à une fraction de classe sociale supérieure. La quête d'un travail socialement valorisé ne laisse pas la place, ici, au renoncement, même si s'estompe parfois l'espoir et pointe l'amertume.

Car cette valorisation du savoir savant opposé au travail profane a un prix : «La société marocaine se divise en catégories, explique Najib : dans le quartier où j'habite, on trouve des gens non cultivés, ils ne m'estiment pas, me disent "tu vas rester tout le temps au chômage" [...]. Alors je me sens comme un étranger dans cette ville, c'est pour ça que je passe tout mon temps loin de ce quartier. Ici c'est différent. Quand tu parles avec les gens, tu sens que ce sont des gens instruits, des gens biens, des gens qui respectent les gens instruits et cultivés qui ont des diplômes. » «Les études apportent beaucoup de choses, mais ça ne suffit pas », précise Saïd. «On souffre et on n'a aucune compensation: la tête, la poche, le corps, tout souffre. Avec cette souffrance, si on ne s'accomplit pas, on finit par être en morceaux. » Mais, on va le voir, le rêve qui les anime et les sauve d'une perception douloureuse du mépris du déclassé, du chômeur, ou de constructions symboliques plus hasardeuses encore, est de croire en leur destin de chercheur.

\section{Ceux qui refusent avant tout le déclassement}

Une vocation scientifique aussi marquée reste en effet le cas d'une minorité. Pour la grande majorité des personnes interrogées, l'obtention du titre universitaire se doit cependant d'être suivie d'un emploi en rapport avec les études, d'une situation — d'un environnement «intellectuel» — où le savoir lui-même trouverait un sens. À moins d'y être contraint par manque total de ressources — « c'est la situation qui t'oblige à faire n'importe quel travail, quand il n'y a pas le choix, il faut relever tes manches et sortir travailler » - , la dévalorisation du diplôme, inhérente à l'exercice d'un travail manuel, est refusée. «Être garçon de café, vendeur ambulant... c'est très difficile d'avoir une licence et de faire ces métiers », estime Abdelkader, licencié en chimie et au chômage depuis un an ; « je ne peux pas les accepter. » Après avoir passé plusieurs concours (d'instituteur, d'infirmier, de technicien), après avoir effectué un stage d'un mois pour devenir formateur en alphabétisation, il attend désormais... L'attente peut être longue (d'autres 
que lui sont au chômage depuis sept, voire neuf ans ${ }^{27}$ ), dès lors qu'elle s'interdit le déclassement consécutif à la pratique d'une activité mal rémunérée ou sans rapport avec le capital acquis sur les bancs de l'université.

Pour nombre de ces jeunes en situation d'inactivité, et en marge dans un milieu où le travail est la voie première de l'accomplissement personnel, le savoir est, d'une certaine manière, tout ce qui reste. L'important, pour eux, n'est alors pas tant le fait que les études se soldent par l'obtention d'un emploi qui leur corresponde parfaitement, mais la possibilité de pratiquer une activité dont ils peuvent tirer un capital social jusque-là interdit. L'acharnement dans la quête de la licence avait cette seule légitimité de procurer de quoi «s'en sortir» durablement dans l'accès à la catégorie sociale supérieure à la leur. Un niveau supérieur d'études ne garantit plus la promotion sociale ; il ne donne plus, non plus, l'assurance d'être reconnu au nom de son savoir par les ignorants, d'être, en quelque sorte, dominant parmi les dominés auxquels on appartient. C'est pourtant la conviction à laquelle chacun s'accroche. L'attente hypothétique d'un travail «convenable », offert par la voie de concours d'entrée dans la fonction publique, est alors préférée. Sous-tendue par la conscience d'une « appartenance refusée » à la classe supérieure, par la conviction que la valorisation du savoir est « sous conditions » (en priorité, la possession de capital social et économique), enfin par la volonté d'échapper à la relégation, cette attente, lorsqu'elle est loisible, permet malgré tout d'échapper à une insertion sociale et professionnelle - en l'occurrence dans le secteur « informel» — à laquelle l'appartenance au milieu populaire prédestine. Plus que la marque de l'échec, la situation de chômeur, ici, est vécue comme une étape, inscrite dans un parcours que le diplôme - dans lequel on veut croire, toujours, et dont on refuse la dépréciation - rendra inéluctablement fructueux. Et, à l'encontre de tout fatalisme, elle demeure le rempart symbolique contre l'échec total et permet de sauvegarder le seul capital monnayable : le savoir et son marqueur principal, le diplôme, dans l'espoir de sa valorisation future et inédite, ici ou ailleurs.

Pourtant, là encore, cette stratégie a un coût en termes d'image de soi dans le regard d'autrui. Comme l'explique Noureddine en évoquant son activité commerciale avec son père : «C'est très dur. Un jeune Marocain qui a passé 18 ans d'études, après, il se trouve à faire du commerce avec des gens qui n'ont pas de culture, des analphabètes. J'ai eu beaucoup de problèmes avec ces gens; ils me blâmaient. Ils me disent: "Tu as étudié et tu as eu un diplôme, pour rien : tu viens vendre des légumes. Tu viens les vendre à côté de nous ; donc nous sommes égaux". Des gens me disent ça. »

27. Entretien avec le président de l'Association des diplômés chômeurs de Fès, mai 2001. 


\section{Et ceux qui travaillent, sans plus d'ambition de carrière}

Après quatre années d'études en faculté des sciences, et après avoir obtenu, en 1997, sa licence d'électronique, Mohamed a fait du « petit commerce » pendant huit mois. Associé à l'un de ses cousins, il achetait des récoltes de fruits et les revendait dans les souks de Fès. Il a tiré de cette expérience une certaine fierté : "L'expérience, c'est ça le but. Je voulais tenter ma chance, sans demander de l'aide à mes parents. » Grâce à un prêt, il a ensuite ouvert une teinturerie dans son quartier, parce qu'il n'a « rien trouvé d'autre ». Ce choix s'est fait par défaut et sans tenir compte de sa qualification, à cause du manque de débouchés en électronique. Qu'importe : «C'est le travail qui donne un sens, qui renforce la personnalité, qui donne ses capacités à l'homme, le rend noble. Pour notre religion, l'homme qui travaille est mieux que celui qui ne travaille pas. » Ses propos laissent penser que la pratique d'un travail qui serait, pour d'autres diplômés, la preuve d'un rabaissement, n'en est pas une pour lui : l'acquisition d'un emploi, la réussite personnelle, s'entourent du respect de l'entourage et lui confèrent toute la reconnaissance qu'il pouvait attendre de sa qualité de diplômé de l'enseignement supérieur. Sa qualification lui permet de se démarquer de ses voisins « ignorants», parmi lesquels il a acquis une position enviable, tirant de son travail toute sa légitimité, sa supériorité : "Ceux qui ont fait des études supérieures et les autres sont différents : même la manière de penser est différente, la culture est différente, la discussion différente, l'avenir n'a pas la même dimension. L'homme qui a été scolarisé est plus fort que celui qui ne l'a pas été. La culture c'est une arme, pour se défendre. » Titulaire d'une licence en chimie organique, Bouazza est, lui, à la tête d'un commerce d'alimentation générale, qu'un prêt contracté auprès de son père et d'un frère lui a permis d'ouvrir. Il n'a aucun regret d'avoir fait des études supérieures ni d'exercer une activité sans aucun rapport avec elles. Au contraire, il est, à ses yeux, «éduqué »; et «les clients me respectent », dit-il. «Sans mes études supérieures, je n'en serais pas là : même des fonctionnaires me demandent, à moi, de leur faire crédit !». Ses clients lui accordent leur confiance grâce à son instruction, signe pour eux de fiabilité et de compétence. Ne le sollicitent-ils pas pour écrire, lire et compter, régler les factures ou calculer l'impôt ? Le souvenir d'avoir fait des études est exempt de toute amertume et est apprécié sous le prisme de la réussite. Le savoir recouvre alors toute sa valeur.

Pour ceux qui partagent cette représentation du travail et du savoir universitaire, l'échec ne réside pas tant dans l'arrêt des études, dans l'abandon d'un doctorat mythique qu'ils pensaient décrocher, que dans l'inactivité, signe à leurs yeux de leur marginalité : "Pour travailler», dit Bouazza, « la question n'est pas celle d'avoir la licence, de choisir un domaine qui vous convient. Il faut travailler, n'importe où [...]. Le chômage, c'est terrible : tu vois tes frères qui travaillent; ils touchent de l'argent et toi tu es assis là, sans argent ; tu manges, tu dors, comme un animal : c'est terrible. » 
Mohamed, Bouazza et Abdelatif choisissent de taire une certaine forme d'échec - la non-appartenance au monde de ceux qui bénéficient d'une ascension sociale sur la base de leur diplôme - et de valoriser au contraire leur réussite dans l'obtention d'un travail et dans la distinction que leur procure leur titre au sein de leurs pairs ${ }^{28}$.

\section{La licence : représentations communes et distinctions}

Les représentations du diplôme tirent ainsi leur substance du statut du travail et des représentations qui lui sont associées. Elles sont à la fois différenciées - selon les individus eux-mêmes et en fonction de leurs situations particulières - et communes : un même sort est partagé, la désillusion de la réussite sociale par la voie universitaire. Tous éprouvent du regret et de l'amertume de ne pas bénéficier d'un travail ni d'un statut à la hauteur des efforts accomplis, de l'ambition d'accéder à une position que leur milieu semble naturellement interdire, mais que le cursus académique avait permis à leurs aînés, après l'indépendance. Le chômage qui frappe nombre d'entre eux prouve à chacun que s'en sortir - sortir de la précarité, sortir d'un milieu socialement dominé — est presque illusoire. Aujourd'hui, le diplôme universitaire est doublement déprécié : non seulement il n'est plus reconnu par l'État ni par le secteur privé qui offre des rémunérations au prorata des licenciés au chômage, et non des savoirs ${ }^{29}$, mais il est également dévalué par les logiques clientélistes mises en œuvre pour progresser dans le cursus universitaire ou pour obtenir un emploi — sous forme d'intervention d'une relation (le «piston », la médiation : wasîta) ou de corruption (rchwa) par l'argent (Bennani-Chraïbi 1994 : 146-148).

\section{Le savoir, agent de distinction «quand même »}

Pour autant, une telle dépréciation ne démotive pas tous ces «non-héritiers » qui s'acharnent à obtenir le titre le plus élevé, à vouloir se doter d'un capital, en l'occurrence scolaire, qui puisse constituer un rempart, à la fois contre l'exclusion et la précarité. On s'accroche à l'idée que «oui, bien sûr, [la licence ne vaut plus grand chose], mais le chômage des aînés n'est pas une fatalité ${ }^{30}$. On ne se résout pas à l'infortune qui guette les «déshérités » du système élitiste de promotion sociale. Pour chacun - et plus encore

28. Ce qui relativise singulièrement l' «adéquation formation-emploi » toujours mise en avant par les politiques pour traiter le cas des "diplômés au chômage », dans l'ignorance du principe de déclassement dont elle se nourrit et qu'elle entretient.

29. Certains licenciés rencontrés sont ainsi rémunérés 750 Dirhams par mois alors qu'un salaire de fonctionnaire correspondant à ce diplôme est de 3300 Dirhams.

30. Les étudiants entrent à l'université sans réellement savoir sur quoi — si ce n'est le titre de licence - peuvent déboucher leurs études, et ont une vision peu réaliste de ce que peuvent faire les lauréats de l'université (SALAHEDINE 1987). 
pour ceux qui sont effectivement en situation d'inactivité ou qui ont définitivement renoncé à toute carrière correspondant à leurs études —, il s'agit en tout état de cause de marquer la valeur supérieure du savoir scientifique, de revendiquer la différence de celui qui sait, d'opposer au statut de travailleur son statut de diplômé. Dépossédés à la base du capital financier et/ou social, qui s'avère indispensable pour forcer l'insertion dans le marché du travail, privés d'emploi ou contraints d'exercer un métier sans rapport avec leur savoir, ils se retrouvent dans une situation d'échec qui implique de porter haut le titre obtenu ; mais, en même temps, cette situation dévalorise aussi le titre : comme le souligne Najib, «nous sommes à la marge, c'està-dire négligés par les autres. Cette connaissance ne peut pas résister dans ce monde ignorant. On ne peut pas réaliser nos rêves ici. C'est très difficile de combattre l'ignorance ». Le sentiment de marginalité accroît l'appartenance aux origines : le savoir est forcé un peu pour mieux insister sur l'identité et la différence et, conjointement, montrer qu'on est victime de cette distinction-là. La valeur énoncée du savoir réside ainsi dans l'épanouissement représenté qu'il procure, le prestige symbolique dont il s'entoure, la distinction dont il s'accompagne. Ahmed, licencié de géographie depuis 1996, nous dit que «la licence, ça vous donne une importance, un intérêt dans la famille. Une valeur très importante, même si on n'a pas de travail. On vous dit: "quand même, tu as obtenu la licence"; l'autre ne l'a pas obtenu ».

Malgré le chômage auquel sont majoritairement promis les lauréats de l'université, le titre apporte une réussite et, de manière inversée - la qualité du «licencié » est appréciée en regard de son savoir et non plus de son inactivité —, le dépassement — non plus le déclassement. L'échec dans le domaine du travail appelle et justifie la survalorisation du titre et de la réussite universitaires. Dépossédés de la possibilité de travailler à la hauteur de leurs attentes, de faire valoir leur savoir, les licenciés rappellent haut et fort les efforts accomplis, l'éducation reçue, le niveau supérieur atteint, pour mieux opposer à la logique d'exclusion, dont ils sont victimes sur le marché du travail, la nécessité de reconnaître encore, et malgré tout, la valeur du savoir accumulé. Faire face au déclassement donne davantage d'importance au titre acquis: le lauréat se dit en marge du monde du travail en raison même de son niveau supérieur — sa situation n'est-elle pas le résultat de l'incapacité des employeurs à proposer à «ceux qui savent» un travail à la mesure de leurs compétences ? C'est pourquoi accepter un travail déqualifié est un choix aussi difficile que celui de maintenir à toute force un projet de carrière qui semble hors de portée. «Les gens qui ne sont pas scolarisés, eux, ne rencontrent pas de problèmes : ils peuvent tout faire, faire n'importe quel travail » estime Rachid, récemment licencié en électronique, «parce qu'ils font partie de cette réalité. Alors ils s'intègrent facilement. Ce qui empêche les licenciés de faire la même chose que ceux qui ne le sont pas, ou qui ne sont pas scolarisés, c'est l'éducation. Par exemple, nous, on connaît plusieurs voies d'éducation, des écoles de pensée... C'est ça qui 
nous empêche d'accepter ce genre de travail : par exemple pompiste, cireur de chaussures, garçon de café, vendeur de cigarettes. »

S'il ne donne pas de droits, le savoir, d'une certaine manière, fait loi. Le diplôme est certes vulgarisé, et les cohortes grossissent d'année en année, mais « le fait d'avoir une licence », explique Mustapha, diplômé en chimie, «ça prouve qu'on est arrivé à quelque chose ». La licence représente le fait d'y être arrivé et l'on veut s'en convaincre, «il y a toujours du respect à l'égard des licenciés, parce qu'ils ont du savoir ».

\section{Savoir et travail, la rupture}

Le refus du déclassement se traduit par l'acharnement à poursuivre des études très poussées lorsqu'il correspond à une volonté de prendre place dans la classe dominante de la société, pour ne pas y occuper une position secondaire. Il se manifeste pour d'autres par la simple ambition d'être employé par l'État, quel que soit le poste proposé. L'acceptation d'un travail dans son propre milieu et sans rapport avec son savoir offre enfin la perspective d'occuper une position supérieure dans son groupe, tout dominé qu'il soit dans la société, et d'échapper ainsi à un type de domination. Les références restent le savoir — en guise de consécration d'un mode historique de domination et la consécration maintenue du savoir universitaire - et le travail - dans le respect du principe moteur de reproduction de son groupe et par refus de souscrire au mode de domination institué sur la base, passée, du savoir et, actuelle, du capital économique.

Dans un cas comme dans l'autre, le savoir et le titre légitiment l'accès à l'emploi, et/ou au statut supérieur dans l'emploi. Quelle que soit leur position, acquise ou subie, les jeunes diplômés d'université refusent la précarité, au nom même des études suivies, et traduisent ce refus en actes.

Telle est bien la figure de cet «entre-deux», formé par la horde des « diplômés chômeurs » réunis en associations ou subsistant dans un «chômage de l'ombre » ignoré des politiques et des médias (Gérard 2002). Cette catégorie - érigée comme telle par les pouvoirs publics ${ }^{31}$ — symbolise en

31. Une cellule ministérielle est notamment chargée de répondre aux manifestations et grèves de la faim des « docteurs et ingénieurs d'État » par des mesures d'intégration. Mais celle-ci n'est pas à même de concentrer les efforts des différents ministères concernés par la question. Le ministre de l'Emploi, de la Formation professionnelle, du Développement social et de la Solidarité, déclarait par exemple, le 17 janvier 2001 et après reprise d'une grève de la faim des jeunes concernés, que «le chômage des docteurs et des ingénieurs est un problème résolu... si seulement les autres ministères concèdent à y participer » (Le Journal hebdomadaire, 27 janvier/2 février 2001, p. 4). En mars de la même année, le gouvernement décidait d'octroyer, «en fonction des postes budgétaires disponibles aux niveaux des secteurs administratifs et des établissements publics » (L'opinion, 18 mars 2001), «1300 postes budgétaires pour l'insertion des diplômés-chômeurs » (ibid., 21 mars 2001). 
elle-même tout le rapport complexe et renégocié entre le travail et le savoir. Elle dévoile aussi, pour peu que soient également interrogées les autres représentations et pratiques des diplômés au chômage ou en activité " informelle », le passage, progressif depuis quinze ans, à un «traitement », social et politique, inédit du savoir universitaire. Contre les politiques universitaires et d'emploi, les étudiants et jeunes diplômés retournent l'argument des réformes : à une « massification » de l'enseignement supérieur dépourvue de moyens adéquats répond l'achat d'inscriptions dans des structures supérieures d'enseignement plus nobles; la sélection accrue est contournée par le passage en fraude des examens par des camarades plus «compétents »; l'achat de diplômes fictifs dans des structures privées d'enseignement ou le recours au piston pallient l'impossibilité de faire valoir son titre sur le marché de l'emploi et la difficulté de poursuivre des études à l'étranger ; en dernier recours, à une dépréciation du diplôme s'opposent des grèves de la faim $^{32}$.

\section{Du champ politique au champ social, le parcours étudiant change de référent}

Auparavant, principe d'élection puis fer de lance du renouveau politique ${ }^{33}$, le savoir universitaire semble désormais sortir de la scène politique et devenir, avant tout, objet de traitement dans, et par, l'espace social : non seulement l'obtention de ce savoir est négociée par ses postulants, mais son utilisation obéit aussi, et de plus en plus, à la primauté du capital social et économique, induisant des stratégies inscrites en marge de l'espace public et politique.

Une lecture du statut du savoir universitaire, à travers les représentations et les stratégies de ses détenteurs, met ainsi au jour l'effet paradoxal d'une massification de l'enseignement, en l'occurrence supérieur: si de plus en plus d'étudiants peuvent y postuler, une proportion moindre, en revanche, peut en tirer un quelconque capital pour travailler ou, simplement, prétendre être intégrée dans son groupe d'appartenance. Une apparente démocratisation masque ainsi des distinctions de classes plus importantes et un type inédit de hiérarchisation entre espaces social et politique.

32. La dernière grève de la faim a été celle des «Docteurs et Ingénieurs d'État » au chômage, en février 2001. Depuis le «mouvement de Salé » de 1991 (BenNANiChrAÏ̈I 1994), l'Association des diplômés inactifs, devenue Association des diplômés chômeurs (ADC) revendique régulièrement l'attribution de postes dans la fonction publique. En 2001, l'Etat rappelait encore sa responsabilité limitée dans la situation des chômeurs et appelait les diplômés à plus d'initiatives en termes de recherche et de création d'emplois. Depuis 1993, de nombreuses mesures gouvernementales ont cherché à relayer l'État et à favoriser cette prise d'initiatives.

33. On pense en particulier aux années 1970 durant lesquelles le Maroc connut ses plus importants mouvements étudiants de contestation et dont sont issues de nombreuses personnalités politiques actuelles. 
Une telle évolution ${ }^{34}$ signale une inversion: si le savoir et le titre ont pu être, à un moment donné, un principe d'acquisition du capital social et économique, non seulement tel n'est plus le cas, mais il semble même qu'ils préludent à un déclassement spécifique, qui constitue le danger principal, celui qui doit être conjuré en priorité : le risque d'une marginalisation prolongée, d'un statut d'éternel mineur, ou de raté qui a mal joué en croyant pouvoir quitter sa classe sociale par l'université. Une minorité de diplômés de l'enseignement supérieur s'autorise encore à s'acharner aux études et à croire dans une mobilité sociale sur cette base ; la majorité, elle, a assimilé le principe du déclassement, choisissant comme pis-aller d'occuper une position à leurs yeux «dominante » dans leur groupe d'appartenance, ou de se livrer à l'infortune des passages aléatoires, parfois usurpés ou clandestins, d'une classe à une autre, ou d'un pays à un autre.

Cette pratique-là signale une évolution inédite et augure d'une transformation accusée du statut et des fonctions du savoir dans la société. Jadis principe de la promotion sociale et de l'accès aux postes politiques, il représente désormais en lui-même un capital social en marge du champ politique. Il semble doter ses titulaires d'une force, sinon de contestation, du moins de changement à la base, bien loin des cercles réservés du pouvoir ${ }^{35}$. Élément d'érection, par le passé, de la classe dominante, et principe de distinction de classes, le savoir vient ainsi complexifier ces différenciations (non plus seulement entre classes mais au sein de chacune d'elles). Peut-il sédimenter à la base, en milieu populaire, un «contre-champ » social, en dehors des chemins tracés par l'élite et en marge du champ politique - ou contre lui ?

Institut de recherche pour le développement, Paris.

34. Celle-ci, rappelons-le, est progressive et a vu ses débuts dès le milieu des années 1980. En 1996, par exemple, une professeur en sciences de l'éducation, économiste, notait que « construire l'université marocaine » consistait à « abolir la dualité d'un système d'enseignement supérieur qui, avec des "facs du cœur" d'un côté et des filières élitistes de l'autre, perpétue un système à plusieurs vitesses qui signifie surtout exclusion pour une grande majorité » (DeBbaGH 1996: 89).

35. En raison même de leur multiplication, les licenciés constituent bien un «problème », voire une force dont les acteurs politiques, au sommet comme à l'échelle locale, doivent tenir compte. On pense par exemple à tel maire d'une grande ville, qui a réservé récemment toute une partie d'un souk municipal en construction aux diplômés chômeurs. À un autre niveau, on peut s'interroger aussi sur les rôles sociaux acquis par cette population diplômée dans son milieu et sur les nouvelles fonctions dont le savoir se trouverait investi à la base. 


\section{BIBLIOGRAPHIE}

\section{ANONYME}

1985 «Enseignement privé : la substitution», Lamalif, $168:$ 25-26.

Antoine, P., Razafindrakoto, M. \& Roubaud, F.

2001 «L'insertion dans trois capitales africaines: Akar, Yaoundé, Antananarivo », in R. Collignon \& M. Diouf (dir.), «Les jeunes : hantise de l'espace public dans les sociétés du Sud ?», Autrepart, $18:$ 17-36.

BAINA, A.

1981 Le système de l'enseignement au Maroc, 3 t., Casablanca, Éditions maghrébines.

Ben Messaoud, M.

1970 «Les raisons de la crise universitaire », Lamalif, $41:$ 48-55.

1972 «L'UNEM face aux politiques », Lamalif, 55 : 7-8.

BENNANI-ChraÏBI, M.

1994 Soumis et rebelles. Les jeunes au Maroc, Paris, CNRS Éditions.

Debbagh, A.

1991 «L'insertion professionnelle des licenciés. Une enquête auprès des stagiaires de la faculté des sciences de l'éducation », Attadriss, 17 : 39-51.

1996 «Coût, financement et rendement de l'enseignement supérieur au Maroc », Annales marocaines d'économie, $16: 81-89$.

El Masslout, A.

1999 La mission inachevée de l'université marocaine, Casablanca, Les Éditions Toubkal.

GEISSER, V. (dir.)

2000 Diplômés maghrébins d'ici et d'ailleurs, Paris, CNRS Éditions.

GÉRARD, É.

2002 «École et devenir au Maroc: regards journalistiques sur l'avenir des diplômés », in É. GÉrARD \& L. Proteau (dir.), «Écoles et "jeunes" dans les médias du Sud», Cahiers de la recherche sur l'Éducation et les Savoirs, $1: 87-111$.

IBAAQUIL, L.

1990 «"L'héritage" culturel et le diplôme comme "position de classe" », Attadriss, $16: 81-103$.

IDRISSI, A.

1981 «Les intellectuels : ambivalence et coupure», Lamalif, 131 : 4-7.

JenAistar, A.

1980 «École, famille et société au Maroc », Lamalif, 116: 49-57. 
KhatiBi, A.

1980 «Qui draine qui ? ", Colloque Le brain drain dans le monde arabe, Beyrouth, 4-8 février 1980, Lamalif, $115: 34-35$.

MounIR, S.

1972 «Esquisse d'une théorie générale de la jeunesse marocaine », Lamalif, 54 : 16-22.

Ouahab, A. \& Noreddine, A.

1980 «L'orientation scolaire et professionnelle : un luxe ou une nécessité », Lamalif, $112: 47-51$.

PASCON, P.

1977 «L'ingénieur entre la logique des choses et celle de classe : critique de la sainte raison technique », Lamalif, $90: 28-33$.

RERHAYE, N.

2001 «Politique à l'Université : le mal vivre estudiantin », Le Journal, 5 (2), 17 au 23 février : 10-11.

RIVET, D.

1999 Le Maroc de Lyautey à Mohammed V. Le double visage du Protectorat, Paris, Denoël.

SALAhEdine, M.

1987 «Appréciation de la formation universitaire par les étudiants titulaires de la licence en sciences », Attadriss, 10.

SALMI, J.

1984 «Les ingénieurs marocains aujourd'hui », Lamalif, 153 : 22-25.

SouAli, M.

1983a «Historique de la réforme de l'enseignement (la réforme entre les discours et les faits) », Lamalif, $145: 32-36$.

1983b «La réforme de l'enseignement supérieur », Lamalif, $146:$ 29-33.

Souali, M. \& Merrouni, M.

1981 «Question de l'enseignement au Maroc», Bulletin économique et social du Maroc, 143-144-145-146.

\section{RÉSUMÉ}

À l'instar de nombreux pays, le Maroc doit faire face aujourd'hui au "chômage intellectuel » de ses diplômés sortis de l'université, voire des écoles d'ingénieurs. Cette situation donne lieu à l'interprétation courante, tant de la part des acteurs politiques que des médias, de "l'inadéquation entre formation et emploi ». Elle pose aussi, et plus profondément, la question de l'articulation entre savoir et travail. À travers l'examen successif de la construction historique de l'université marocaine, 
puis des représentations du diplôme par les lauréats de l'enseignement supérieur, issus du milieu populaire, enfin de leurs pratiques d'intégration, cet article montre que, après avoir été au centre du principe d'acquisition du capital social et économique, le savoir et le titre préludent aujourd'hui au déclassement. Ils génèrent en outre la conquête de positions dans sa classe, dominée, d'appartenance — plutôt que de chercher à accéder à la classe supérieure —, ou la décision de s'exiler à l'étranger. En conclusion, cet article avance l'hypothèse que le savoir universitaire pourrait sédimenter un "contre-champ » social, en marge de l'élite et du champ politique.

\section{ABSTRACT}

The Short-comings of Knowledge: Ideas about Diplomas and Work in Morocco. Like many other countries, Morocco has to cope with joblessness among young people with degrees from universities or even engineering schools. Politicians as well as journalists often interpret this "intellectual joblessness" as proof of the lack of a fit between education and the job market. At a deeper level, how is knowledge linked to work? The history of Moroccan universities, the ideas that postsecondary students from the lower classes have about diplomas, and integration practices successively come under examination. After opening the way toward acquiring social and economic "capital", education and diplomas now lead to a loss of status. Furthermore, they motivate actions for acquiring positions in the student dominated social class (rather than obtaining access to a higher class) or decisions to go abroad. The hypothesis is advanced that a higher education might "sediment a social counterfield" on the outskirts of the elite and the political realm.

Mots-clés/Keywords : Maroc, capital social, diplôme, "jeunes diplômés », mobilité sociale, représentations, savoir universitaire, Université/Morocco, social capital, diplomas, young graduates, social mobility, higher education, university. 